\title{
Zeeman-Doppler imaging: old problems and new methods
}

\author{
Thorsten A. Carroll, Markus Kopf, Klaus G. Strassmeier \\ and Ilya Ilyin
}

Astrophysikalisches Institut Potsdam, An der Sternwarte 16, D-14482 Potsdam, Germany email: tcarroll@aip.de

\begin{abstract}
Zeeman-Doppler Imaging (ZDI) is a powerful inversion method to reconstruct stellar magnetic surface fields. The reconstruction process is usually solved by translating the inverse problem into a regularized least-square or optimization problem. In this contribution we will emphasize that ZDI is an inherent non-linear problem and the corresponding regularized optimization is, like many non-linear problems, potentially prone to local minima. We show how this problem will be exacerbated by using an inadequate forward model. To facilitate a more consistent full radiative transfer driven approach to ZDI we describe a two-stage strategy that consist of a principal component analysis (PCA) based line profile reconstruction and a fast approximate polarized radiative transfer method to synthesize local Stokes profiles. Moreover, we introduce a novel statistical inversion method based on artificial neural networks (ANN) which provide a fast calculation of a first guess model and allows to incorporate better physical constraints into the inversion process.
\end{abstract}

Keywords. Stars: activity - stars: magnetic fields - stars: spots - radiative transfer - methods: data analysis

\section{Introduction}

Zeeman-Doppler Imaging (ZDI) was pioneered by Semel (1989), although first described as a mere detection method for surface magnetic fields on rapid rotating stars, the method quickly evolved to a real mapping (inversion) technique (Donati et al. 1990; Brown et al. 1991). Today ZDI or Magnetic-Doppler Imaging (MDI) is a synonym for the inversion of time and phase resolved spectropolarimetric observations to reconstruct stellar magnetic surface fields. Since that time ZDI was a success story of its own and has enormously contributed to our current understanding of stellar magnetism (e.g. Donati 1999; Hussain et al. 2002; Kochukhov et al. 2004; Donati et al. 2006, 2007; Petit et al. 2008). However, there are a number of fundamental problems which has to be considered when dealing with ZDI as an inverse problem. One of these problems comes from an inadequate formulation and approximation of the forward problem while another comes from the inherent non-linearity of the inverse problem.

\section{The forward problem}

Inverse problems arise whenever one searches for causes of observed data or desired effects. The most general way of solving the inverse problem (directly or indirectly) is to utilize a model of the underlying forward problem. The forward problem constitutes our physical theory and/or knowledge about the system to make predictions about the outcome and result of measurements. The direct or forward problem in ZDI is typically the polarized radiative transfer based on a model of the stellar atmosphere. Here we may encounter the first fundamental problem, owing to our limited theoretical knowledge (e.g. 
lack of atomic and/or atmospheric parameters) as well as numerical and computational constraints we are restricted to an approximate description of the real physical system. Critical in this context is to provide an appropriate and adequate parameterization of the model, i.e the minimal number of free variable that determine the measurable outcome. The determination of a particular parameterization is not always obvious and requires a careful evaluation. Formally the forward problem of ZDI can be written in a compact way, by using the formal solution of the Stokes vector as described by Landi Deglinnocenti \& Landi Deglinnocenti (1985). This allows us to write the disk-integrated Stokes vector $\boldsymbol{I}^{*}$ at a wavelength $\lambda$ and for a particular rotational phase $\phi$ as follows :

$$
\boldsymbol{I}^{*}(\lambda, \phi)=\int_{\hat{M}} \int_{0}^{\infty} \boldsymbol{O}\left(0, s\left(M^{\prime}, \theta\right), \boldsymbol{X}\left(M^{\prime}\right), M^{\prime}, \phi\right) \boldsymbol{j}\left(s\left(M^{\prime}, \theta\right), \boldsymbol{X}\left(M^{\prime}\right), M^{\prime}, \phi\right) d s \cos \theta d M .
$$

The vector $\boldsymbol{X}$ provides the parameterization of the model and comprises parameters like the magnetic field vector, temperature, abundance, etc., $d M$ denotes the infinitesimal surface element at the position $M^{\prime}$ (given in spherical coordinates), $\theta$ denotes the angle between the surface normal at $M^{\prime}$ and the direction to the observer, $s$ is the geometrical path length towards the observer, $\boldsymbol{j}$ is the emission vector (see Stenflo 1994), and $\boldsymbol{O}$ is the evolution operator of the formal solution which incorporates the absorption matrix. Following Landi Deglinnocenti \& Landi Deglinnocenti (1985), we can write the evolution operator, in the case of a piecewise constant absorption matrix $\boldsymbol{K}$, as

$$
\boldsymbol{O}\left(s, s^{\prime}, M, \boldsymbol{X}(M)\right)=e^{-\boldsymbol{K}(M, \boldsymbol{X}(M))\left|s^{\prime}-s\right|} .
$$

Equation 2.1 can also be written symbolically in a more compact form as

$$
\boldsymbol{I}^{*}(\lambda, \phi)=\boldsymbol{\Omega}(\boldsymbol{X}),
$$

where $\boldsymbol{\Omega}$ represents the formal non-linear integral operator of the forward problem. Several things must be kept in mind here, both integrals, the outer surface integration over the model atmosphere as well as the inner transport integral through the atmosphere, must be discretized in order to cope with the problem numerically. We should be aware that this already means that we transform our originally continuous problem into a discrete one with finite dimension. As mentioned above, the finite parameterization of our model $\boldsymbol{X}(M)$ deserves particular attention because the limitation to those variables that we consider as necessary and sufficient, will directly determine the dimension of the model space, we will see later how the neglect of the temperature may have drastic consequences for the inversion. The radiative transfer model we use is still formulated in local thermodynamic equilibrium (LTE) which is quite sufficient for the majority of photospheric spectral lines we are using but certainly has its limitations. Moreover, it is customary to describe the atmosphere as height independent, i.e. neglecting all height gradients in most of the model parameter, an approximation which becomes questionable in particular for stars with extended atmospheres like giants. All these approximations of the real physical situation in stellar atmospheres will therefore have direct consequences on the inversion process. Finally, it should be also clear from equation (2.1) and (2.2) that our forward problem is in general non-linear in $\boldsymbol{X}$, an aspect that will be discussed further in the following section.

\section{The inverse problem}

The retrieval of model parameters from measured data constitutes the general definition of a inverse problem. In the particular case of ZDI the measured data are given in 
the form of phase resolved Stokes profile observations. In general there is a distinction between continuous and discrete inverse problems which means that we want to retrieve either a continuous functions or a parameterized model. In that sense it is already the formulation of the forward problem which defines the kind of inverse problem. The same holds for the definition of linear or non-linear inverse problems, which is determined by the linearity or non-linearity of the forward problem. Inverse problems, like ZDI, are often said to be ill-posed by which, in general, we mean that i.) the inverse problem might be non-unique and ii.) the inverse problem might not depend continuously on the data, i.e. the solution is unstable against small perturbation of the observed data. The non-uniqueness may have different reasons which can be a real intrinsic non-uniqueness of the problem, or uncertainties in the observed data, or an ill-defined forward model, or a lack of data that sufficiently constrain the problem. The problem of ill-posedness can be effectively addressed by a regularization approach, i.e. adding additional a priori assumptions (i.e. constraints) to the inverse operator. A powerful method in this context is the Tikhonov regularization but many other forms of regularizations are also possible. For linear problems it can indeed be shown that the regularization leads to a unique and stable solution (see e.g. Engl et al. 1996). However, the forward problem we deal with in ZDI is non-linear, and at first, it is not obvious how this will affect the uniqueness and stability of our solution. The general way to proceed is to formulate and minimize a regularized least-square problem. This approach, relies on the linearization of the forward operator, which can be obtained in our case from a first order expansion of (2.3),

$$
\boldsymbol{\Omega}(\boldsymbol{X})=\boldsymbol{\Omega}\left(\boldsymbol{X}_{0}\right)+\boldsymbol{J}\left(\boldsymbol{X}-\boldsymbol{X}_{\mathbf{0}}\right)
$$

where $\boldsymbol{J}$ is the Jacobian matrix of $\boldsymbol{\Omega}$. Using the sum of square error and a regularization functional $G(\boldsymbol{X})$ the objective functional $F(\boldsymbol{X})$ can be written as

$$
F(\boldsymbol{X})=\left\|\boldsymbol{I}_{o b s}-\boldsymbol{I}^{*}\right\|^{2}+\alpha G(\boldsymbol{X})
$$

where $\alpha$ is the regularization parameter which determines the influence of the regularization. The regularization functional $G$ can be expressed in the from $\left\|L\left(\boldsymbol{X}_{\text {ref }}-\boldsymbol{X}_{0}\right)\right\|^{2}$ where $\boldsymbol{X}_{r e f}$ is a parameter vector which represents our a priori knowledge (assumption) about the solution, and $L$ is a suitable matrix approximation of a differential operator. The essence of the non-linear solution is then to repeat the process of solving the linearized solution in terms of a gradient descent method. Using a Gauss-Newton type form of linearization (Engl et al. 1996) we obtain from (3.2) for an increment $\Delta \boldsymbol{X}_{n+1}$ at the iteration $n+1$ the following solution,

$$
\Delta \boldsymbol{X}_{n+1}=\left(\boldsymbol{J}^{*} \boldsymbol{J}+\alpha \boldsymbol{L}^{*} \boldsymbol{L}\right)^{-1}\left(\boldsymbol{J}^{*}\left(\boldsymbol{I}_{o b s}-\boldsymbol{I}^{*}\right)\right)+\alpha \boldsymbol{L}^{*} \boldsymbol{L}\left(\boldsymbol{X}_{r e f}-\boldsymbol{X}_{n}\right),
$$

where $\boldsymbol{J}^{*}$ is the conjugate transpose of $\boldsymbol{J}$. The stabilizing effect of the regularized solution is then ensured by the positive definiteness of the inverse $\left(\boldsymbol{J}^{*} \boldsymbol{J}+\alpha \boldsymbol{L}^{*} \boldsymbol{L}\right)^{-1}$. As the regularization parameter is held constant throughout the iteration, the regularization part in (3.2) will have an increasing influence as we approach the solution and thus provides the additional benefit of preventing the inversion from fitting arbitrary details (i.e. noise) in the spectra. However, although we gain a stabilizing effect at each iteration step, it should be clear from (3.2) that even when $G$ provides a convex constraint this needs not necessarily be true for $F(\boldsymbol{X})$ which, in general, is still a non-convex function due to the non-linearity of (2.1). The inverse problem may therefore have many local minima in the parameter space in which a gradient descent method can get stuck and a regularization alone can not guarantee to find the unique global minimum. Furthermore, the determination of an appropriate regularization parameter $\alpha$ is by no means an easy task and can require high computational efforts (Engl et al. 1996). 

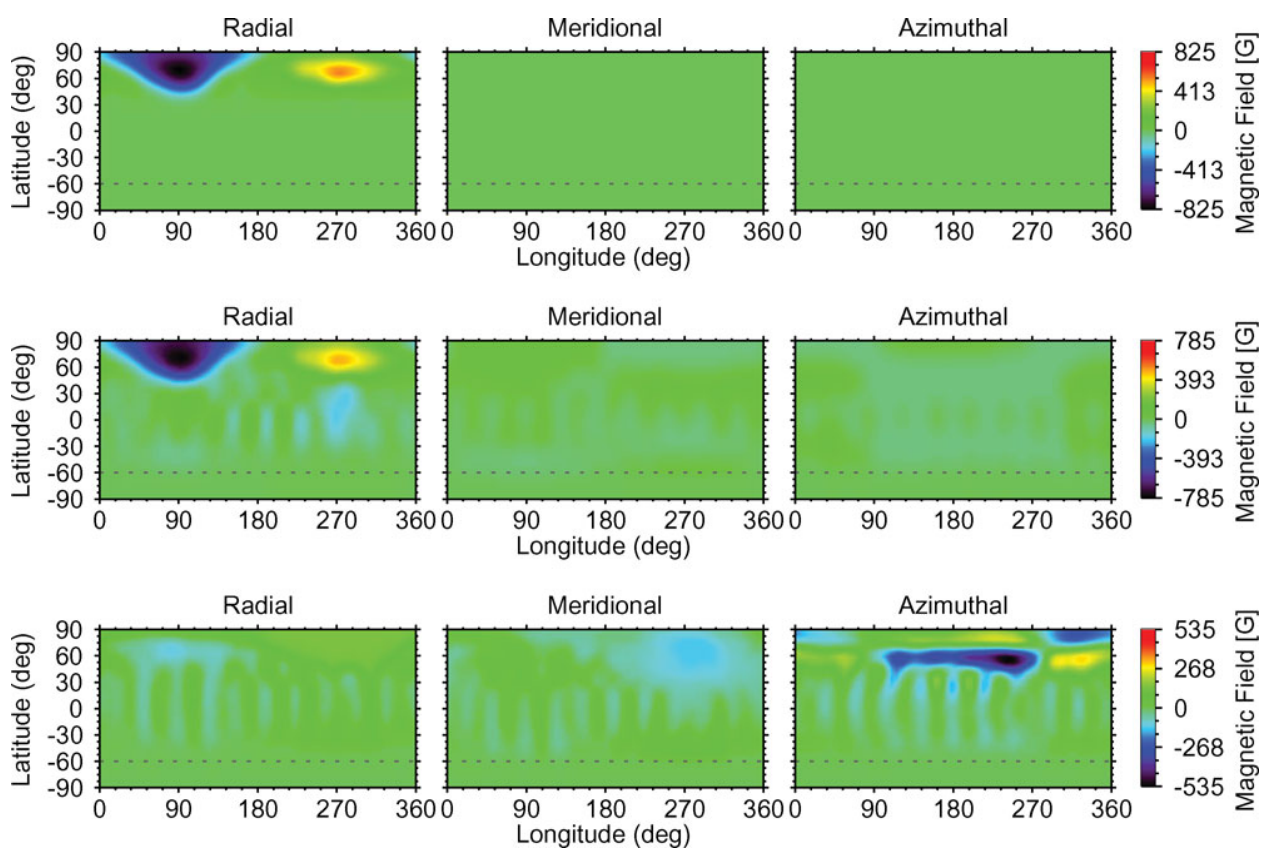

Figure 1. Mercator plots of a simulated magnetic field distribution. Top: the synthetic reference model. Middle: the reconstructed magnetic field from a simultaneous temperature and magnetic field inversion. Bottom: the result from the restricted inversion (fixed temperature). See text.

\section{More than one solution - an inadequate forward model}

To show how much the solution of the ZDI problem depends on the forward model and the chosen parameterization we made a simple synthetic experiment. The stellar model surface consists of a large bipolar magnetic field region. The stellar atmosphere is that of a typical fast rotating $\mathrm{K}$ subgiant star (i.e. $\log (g)=3.5, T_{\text {eff }}=4600 \mathrm{~K},[\mathrm{M} / \mathrm{H}]$ $=-0.5, \mathrm{vsini}=40 \mathrm{~km} / \mathrm{s}$ ). One of the magnetic regions is located in a cool spot like structure while the other polarity is embedded in a hot and more dispersed like structure to mimic a faculae or plage region, see top of Figure 2 The magnetic field is exclusively radial oriented in both magnetic regions. Two inversion were performed, the first one worked on the basis of the correct parameterization with the magnetic field vector and temperature as free parameter, while the second inversion runs with a fixed temperature. This allows the second inversion only to seek in a subspace for a possible solution. For the inversion we have our ZDI code iMap (Carroll et al. 2007) with a surface segmentation of 1800 elements, and the Zeeman-sensitive iron line FeI $\lambda$ 6173. Although we have all four Stokes components available we restricted the number of available Stokes components to Stokes $I$ and Stokes $V$ of the Zeeman-sensitive iron line FeI $\lambda$ 6173. We have added noise with a signal-to-noise level of 1000 to the original model spectra. Both inversion run with the same value for the regularization parameter. The first inversion converged to a solution that is close to the reference field structure, see Figure 1 middle. The surface structure is well reconstructed from the simultaneous temperature and magnetic field inversion and also the Stokes $V$ spectra (Figure 2, left) are well reproduced. In the second inversion where we have neglected the temperature as a free parameter the fit to the reference spectra is again very good but the resulting magnetic surface field is drastically different (Figure 1, bottom). The surface field shows a characteristic high 

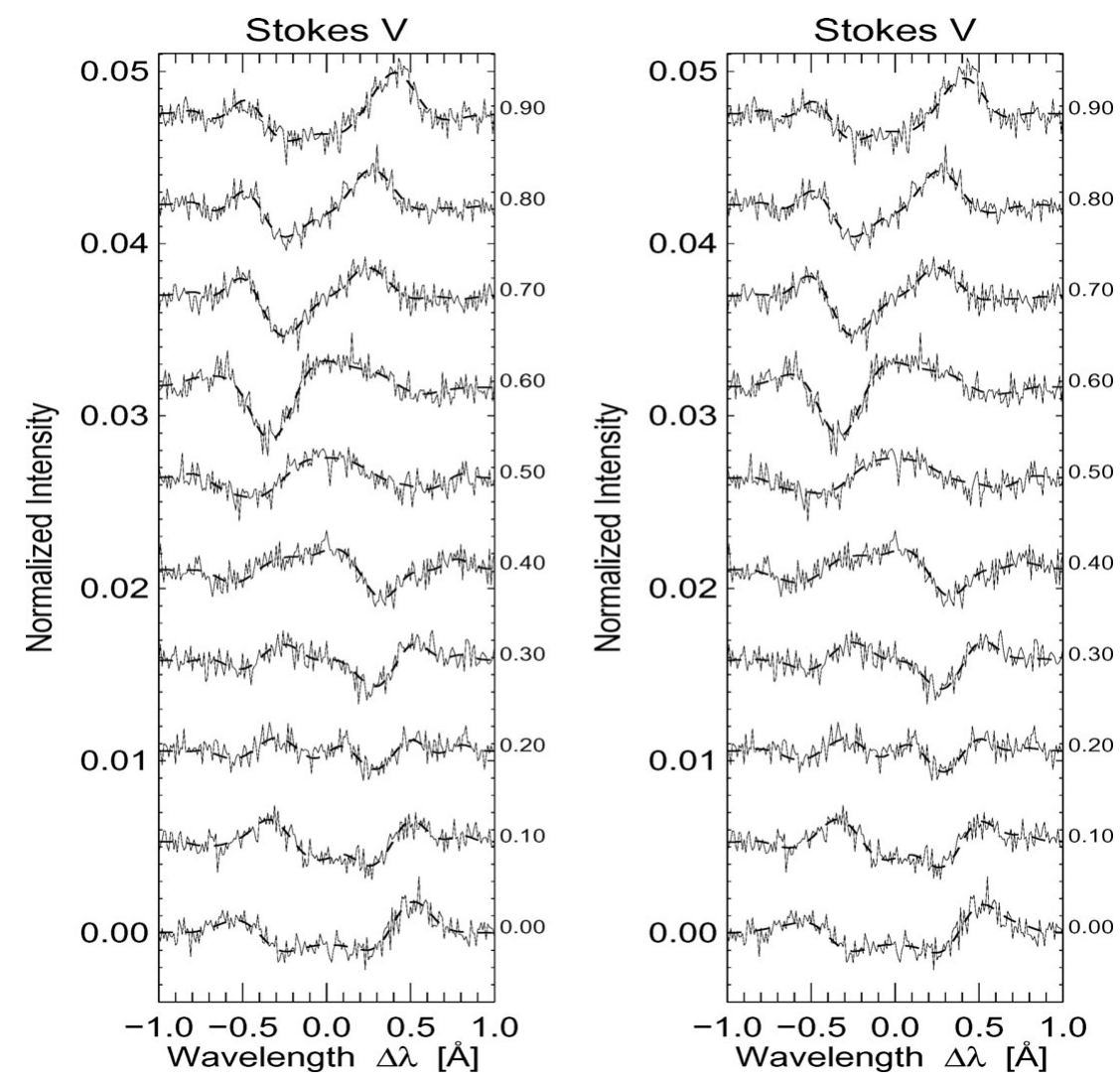

Figure 2. The fits (dashed lines) to the noisy reference Stokes $V$ profiles from the two test inversions : on the left the fits of the simultaneous temperature and magnetic field inversion, $\chi_{\text {red }}^{2}=1.01$ and on the right the fits of the restricted inversion, $\chi_{\text {red }}^{2}=1.02$.

latitude band of a unipolar azimuthal field with an longitudinal extension of about $180^{0}$ which is in sharp contrast to the original reference field. The inversion in the restricted parameter regime has apparently converged to a false solution, although the fits are very good (Figure 2, right). The quality (goodness) of the fit (Figure 2) itself do not provide enough information in this case to distinguish between the competing solutions. This issue could readily be resolved if the corresponding Stokes $Q$ and $U$ profiles are taken into account (not shown here). Due to the critical role of the temperature and the associated radiative transfer effects it will be of particular importance to provide an accurate and appropriate forward model for the inverse problem. We will investigate the impact of radiative transfer effects and inadequate modeling for ZDI inversions in greater detail in a forthcoming paper (Carroll et al. 2009).

\section{Towards a full polarized radiative transfer approach}

We have seen from the foregoing synthetic example how the underlying model can affect the outcome of the inversion. Moreover we have seen the critical role of the temperature which is of course tightly correlated to the radiative transfer effects. It is therefore worthwhile to include a full polarized radiative transfer in the inversion process to make the 
problem setting as realistic as possible. There are at least two major obstacles towards a full radiative transfer driven approach :

- the noise level of individual Stokes line profiles, and

- the computational demands of an iterative inversion.

The first point refers to the cases that most observed and measured Stokes $V, Q$ and $U$ spectra are heavily contaminated by noise. Although there exist powerful multi-line reconstruction techniques as the least-square deconvolution method of Donati et al. (1997), they do not easily allow one to apply a radiative transfer modeling approach. The crucial point is that this technique only retrieve a kind of mean line profile which has no straight forward interpretation on the basis of spectral line synthesis. The second point highlights the computational effort one is facing by the iterative approach to the inverse problem. To address both of the above listed problems and to pave the way for a full radiative transfer modeling approach we propose a two-step strategy, a multi-line reconstruction method which enables the reconstruction of individual Stokes profiles and a fast approximate radiative transfer calculation by artificial neural networks.

\subsection{Multi-Line PCA reconstruction}

The basic idea of the multi-line PCA reconstruction technique is to extract the systematic features within the observed data set, i.e. Stokes profiles, and at the same time to reduce the noise. This is done by identifying the redundancy within the dataset and describing the dataset with a minimal number of characteristic parameter (i.e. dimensions) as possible. The redundancy among the observed data can be described by the correlation between the individual spectra such that we can express the correlation of entire observational dataset by the covariance matrix $\boldsymbol{C}_{x}$ which can be written, after transforming the spectra into the velocity domain, as

$$
\boldsymbol{C}_{x}=\sum_{n}\left(\boldsymbol{X}_{n}(v)-\overline{\boldsymbol{X}}(v)\right)\left(\boldsymbol{X}_{n}(v)-\overline{\boldsymbol{X}}(v)\right)^{T},
$$

where $v=c \Delta \lambda / \lambda$ and $n$ is the number of individual spectral line profiles used for the analysis and $\overline{\boldsymbol{X}}$ the mean Stokes profiles of all spectral lines. We then seek a set of orthonormal direction in the data space (given by the spectral resolution) along which the variance in $\boldsymbol{X}$ is maximized in descending order. These new set of coordinate axes which accounts for the maximum variance in the observed data (Stokes spectra) can be determined by calculating the eigenvectors of the covariance matrix Eq. (5.1). The so called Principal Component Analysis (PCA) or Karhunen-Loeve transformation (Bishop 1995) will provide us with the calculation of the eigenvectors of the covariance matrix of the observation whereby the eigenvectors are ordered according to their associated eigenvalues (variances). We use the PCA method to decompose the entire set of observed Stokes spectra into a new coordinate system. This procedure will project the most coherent and systematic features in the observed Stokes profiles into the first few eigenvectors with the largest eigenvalues while the incoherent features (i.e. noise or blends) will be mapped to the less significant eigenvectors (with low eigenvalues). After having calculated the set of orthogonal eigenvectors we can use this new basis to decompose all observed Stokes spectra into the new basis of eigenvectors $\boldsymbol{u}_{\boldsymbol{l}}$, as

$$
\boldsymbol{x}_{k}(v)=\sum_{l} \alpha_{k, l} \boldsymbol{u}_{l}(v)
$$

where $\alpha_{k, l}=\boldsymbol{x}_{k}(v) \boldsymbol{u}_{l}(v)$ is the scalar product (i.e. the projection or the cross-correlation) between the observed $k$-th Stokes profile $\boldsymbol{x}_{k}(v)$ and the $l$-th eigenvector $\boldsymbol{u}_{l}(v)$. If we use a limited number of the first few $l$ eigenvectors for this decomposition the reconstruction 
will be made only with those components who carry the most significant information about the systematic features and inherent line characteristics. We therefore reconstruct individual Stokes profiles to an extent that the majority of the individual line characteristics are preserved and only a small contribution of uncorrelated effects are present. To determine the maximum number of meaningful eigenvectors we calculate the covariance matrix of pure noise profiles with a $\mathrm{S} / \mathrm{N}$ ratio that corresponds to the noise level of the observations. This allows us to estimate the magnitude of the eigenvalues from which the corresponding eigenvectors will mainly contain noise. Since the PCA multi-line method now allows the reconstruction of individual line profiles, all the known line parameters of a particular spectral line can be used in a subsequent DI and ZDI inversion. See Carroll et al. (2007); Martínez González et al. (2008) for a more detailed description of the method.

\subsection{A fast Stokes profile synthesis}

The basic idea of our proposed approach is to emulate the process of polarized line formation by using an adaptive regression model, that is fast to evaluate, and which provides the required accuracy. The adaptive model we seek must provide a sufficient complexity to describe the non-linear mapping of Eq. (2.1), between the most prominent atmospheric parameters and the resulting Stokes spectra. For this purpose, we used a supervised machine learning algorithm, e.g., an artificial neural network (ANN) model. A popular type of of ANN, also used in this work, is the so called multilayer-perceptron (MLP) which is known as a universal function approximator. The MLP can be regarded as a class of nonlinear function, which performs a continuous and multivariate mapping between an input vector $\boldsymbol{x}$ and an output vector $\boldsymbol{y}$. The network function represents a function composition of elementary non-linear functions $g(a)$. These elementary functions are arranged in layers whereby each of these functions in one layer is connected via an adaptive weight vector $\boldsymbol{w}$ to all elementary functions in the neighboring layers. The $l-t h$ output (component of the output vector $\boldsymbol{y}$ ) of a two layer (of weights) MLP for example can be written as

$$
y_{l}(\boldsymbol{x} ; \boldsymbol{w})=g_{k}\left(\sum_{j=0}^{J} w_{k j}^{(2)} g_{j}\left(\sum_{i=0}^{I} w_{j i}^{(1)} x_{i}\right)\right),
$$

where $x_{i}$ represents the $i$-th component of the input vector $\boldsymbol{x}$ and $w_{j i}^{(1)}$ the connecting weight from the $i$-th input component to the $j$-th elementary function $g_{j}$ in the first unit layer. The weights $w_{k j}^{(2)}$ then connecting all the functions $g_{j}$ with the functions $g_{k}$ in the second unit layer. The capital letters $(\mathrm{I}, \mathrm{J})$ giving the numbers of elementary functions (units) in the respective unit layer. The elementary functions $g(a)$, which are also called activation functions, are given by the following type of sigmoid function,

$$
g(a)=\frac{1}{1+\exp (-a)} .
$$

The network function $\boldsymbol{y}(\boldsymbol{x})$ will thus process a given input vector $\boldsymbol{x}$ by propagating this vector (via multiplication with the individual weight values and subsequent evaluation of the different activation functions) through each layer of the network. The particular function that will be implemented by the MLP is determined by the overall structure of the network and the individual adaptive weight values. The process of determining these weight values for the MLP is called (supervised) training and is formulated as a non-linear optimization process. This training is performed on the basis of a representative dataset, which includes the input to target (i.e. training output) relations of the underlying problem. This process is similar to a non-linear regression for a given 
data set, but as the underlying model (i.e., MLP) is much more general, the regression function is not restricted to a specific predetermined (or anticipated) model. In fact, it can be shown that MLPs provide a general framework for approximating arbitrary non-linear functions (Bishop 1995). In our case the input-to-target relation is dictated by our synthesis problem, for the input vectors we have chosen the following atmospheric parameters : the temperature and pressure structure of model atmospheres (described by the effective temperature), logarithmic gravitation, iron abundance, the local bulk velocity of the plasma, microturbulence, macroturbulence, magnetic field strength, magnetic field inclination, magnetic field azimuth and the LOS angle between the observer and the local normal. For the output parameter we have used the full Stokes vector profiles of the Zeeman-sensitive iron lines FeI $\lambda 6173 \AA$ and FeI $\lambda 5497 \AA$ which have an effective Landé factor of $g_{\mathrm{eff}}=2.5$ and $g_{\mathrm{eff}}=2.25$ respectively. Once the network is successfully trained and has converged in terms of minimizing the error between the calculated output vectors and the target vectors of the training database, the network weights are frozen and the MLP, which now represents the desired approximation of the underlying problem, can be applied to new and unknown data coming from the same parameter domain as the training data. To test the accuracy of the trained MLPs we created a large number of input vectors with randomly chosen combinations for the atmospheric parameters. The statistical evaluation shows that the MLPs are able to calculate the corresponding Stokes profiles with a high degree of accuracy. The comparison of the MLP calculation with the results from the conventional numerical integration of the polarized radiative transfer, performed with the DELO method of Rees et al. (1989), demonstrates the impressive results of the MLP synthesis. For the Stokes $I$ profile calculation we obtain a rms error as low as $0.11 \%$, for Stokes $V 0.17 \%$; and for Stokes $Q$ and $U$, slightly above $1 \%$ relative to the DELO solution. The MLP has in fact learned to disentangle the different and sometimes competitive effects of the various atmospheric input parameters to calculate accurate local Stokes profiles for both iron lines. But it is not only the accuracy of the method which makes this synthesis method attractive for ZDI inversion, it is first and foremost the speed of the calculation. A benchmark test with several workstations confirmed that the MLP synthesis is more than a factor 1000 faster then the conventional numerical approach, for more details see Carroll et al. (2008).

\section{An artificial neural network approach to ZDI}

In this section we want to introduce our new statistical inverse approach to ZDI which is based on artificial neural networks. Instead of using the ANNs in a forward modeling approach as in the preceding section, we now try to use ANNs to provide a direct approximation of the inverse mapping between the observed Stokes spectra and the corresponding magnetic surface distribution. Again we rely on the non-linear approximation capabilities of the multilayer-perceptron as given in Eq. 5.3, to find a continuous approximation of the underlying inverse problem. ANNs have been already successfully used in solar Stokes profile inversion problems (Carroll \& Staude 2001; Carroll \& Kopf 2008) which have shown their great potentials in approximating the inverse mappings between observed Stokes profiles and the underlying atmospheric parameters. One of the key issues in the following ZDI inversion is the reduction of the input (i.e. Stokes spectra) as well as the output dimension (i.e. surface images) by a PCA decomposition. The learning task of the neural network is then to approximate the inverse mapping in the reduced eigenspace between the eigenprofiles and the corresponding eigenimages of the surface. The reduction to the subspace of eigenspectra and images has two main reasons, first, we reduce the topology of the MLP structure which allows a more stable and faster 

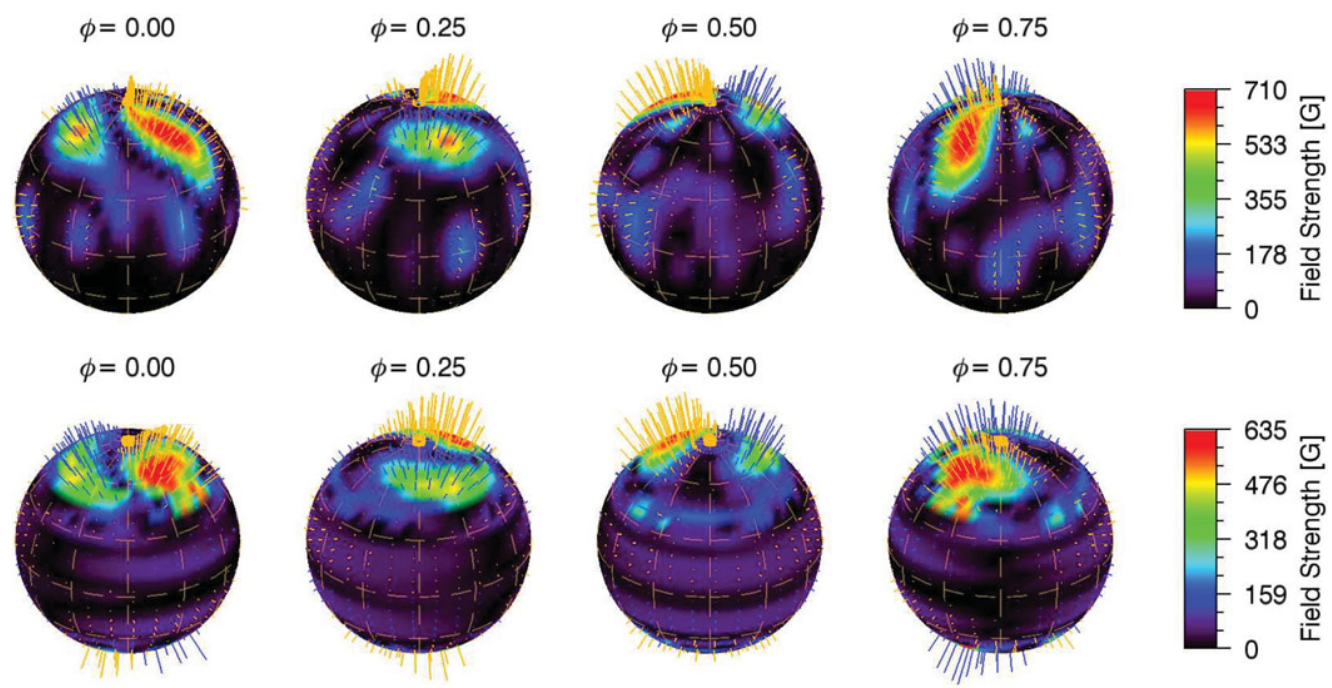

Figure 3. The reconstructed radial magnetic field of II Peg for the year 2007. In the top the reconstruction based on the conventional inversion is shown, whereas, the ANN inversion based on the $\alpha^{2}-\Omega$ dynamo model is shown in the bottom.

convergence in the training process and second, the restriction to a subspace of the problem, has a regularizing effect on the solution due to the reduced redundancy in the input spectra and the noise reducing effects by describing the solution as a superposition of eigenimages. Because the transformation and training of the network is based upon a training sample, this will have a decisive impact on the resulting eigenspectra and eigenimages. A finite training sample will only provide a good statistical modeling in a limited region of the parameter domain and an approximation of the inverse function will therefore only provide meaningful results in that restricted domain. To apply the ANN inversion on real observed Stokes $V$ spectra (Carroll et al. 2007) we trained our MLP on synthesized Stokes profiles which are based on a theoretical $\alpha^{2}-\Omega$ dynamo (Elstner \& Korhonen 2005). The $\alpha^{2}-\Omega$ dynamo originally developed to study and explain the appearance of flip-flop events on active stars provides not only a good model representation since our target star II Pegasi is known to be a candidate for a flip-flop cycle (Berdyugina 2007), it also allows us to incorporate (implicitly) the physics provided by this model into our inversion approach. From this simulation we have synthesized approximately 1000 Stokes $V$ profiles at different phases and evolution stages of the dynamo. Assuming typical stellar and atmospheric parameters for the subgiant K2 star II Peg (Berdyugina et al. 1998) we have synthesized approximately 1000 disk-integrated Stokes $V$ profiles for different phases and different evolution stages of the dynamo simulation. The training process follows again a large-scale optimization of the network parameters of the MLP to find the best possible approximation for the inverse mapping between the decomposed Stokes $V$ profiles and the corresponding decomposed surface images After the training process the observed II Peg Stokes $V$ spectra applied to the trained MLP to estimate the magnetic surface structure. In Figure 3 we see a comparison of the surface magnetic field structure of II Peg retrieved with the conventional ZDI inversion made with our ZDI code $i M a p$ and the new ANN inversion. The surface structure inferred from this first ANN approach is in remarkable good agreement with the conventional inversion. The good convergence of the training process and the good results of the inversion indicate that the MLP has in fact found a well-behaved inverse mapping to that problem and moreover 
it shows that the dynamo model used for this study provides a reasonable assumption for the surface structure observed on II Peg. A more detailed description of this novel ANN approach will be given in a forthcoming paper (Carroll et al. 2009).

\section{Summary}

Zeeman-Doppler imaging is an inherent non-linear problem even with an appropriate regularization, it is not assured that we arrive at a global minimum, we are still facing the problem of local minima. This is particular problematic in combination with an ill-defined or inadequate forward model. As was shown in Sect. 4, even if the observed spectra are well reproduced, the solution can be far from the true solution. The fit to the observed Stokes $V$ profiles does often not provide enough information to distinguish between competing solutions. The additional availability of Stokes $Q$ and $U$ profiles with the next generation of spectropolarimeter (PEPSI) at the $8.4 \mathrm{~m}$ Large Binocular Telescope (LBT) (Strassmeier et al. 2007, 2008) will immensely help to further mitigate this problem. But for all the current observations where only Stokes $V$ spectra are available we have to rely on the best possible forward modeling. In an effort to provide a more systematic radiative transfer modeling we have introduced a new PCA based multi-line reconstruction technique. This method allows us to recover Stokes profiles of individual spectra lines and thus facilitates their modeling by means of radiative transfer calculations. Moreover, we have introduced a fast Stokes profile synthesis which is not only accurate but accelerates the process of calculating the Stokes profiles by more than three orders of magnitude. In a preliminary study we have investigated the capabilities of a new artificial neural network approach to ZDI. ANNs have a number of favorable features, they provide a direct inversion in terms of approximating the inverse mapping between the observed Stokes profiles and the magnetic surface distribution. And moreover, ANNs as a statistical learning tool, allows one to incorporate theory based knowledge in the training process which is then utilized in the subsequent inversion. Although further investigations are needed, the ANN approach already provides a new and promising way of a direct inversion or for a hybrid approach with a conventional ZDI inversion.

\section{References}

Berdyugina, S. V. 2007, Mem. Soc. Astr. It. 78, 242

Berdyugina, S. V., Jankov, S., Ilyin, I., Tuominen, I., \& Fekel, F. C. 1998, A\&A 334, 863

Bishop, C. M., 1995, Neural Networks for Pattern Recognition, Oxford University Press

Brown, S. F., Donati, J.-F., Rees, D. E., \& Semel, M. 1991, A\&A 250, 463

Carroll, T. A., Kopf, M., Ilyin, I., \& Strassmeier, K. G. 2009, A\&\&A, in prep.

Carroll, T. A. \& Kopf, M. 2008, A\&A 481, L37

Carroll, T. A., Kopf, M., \& Strassmeier, K. G. 2008, A\& $A$ 488, 781

Carroll, T. A., Kopf, M., Ilyin, I., \& Strassmeier, K. G. 2007, AN 328, 1043

Carroll, T. A. \& Staude, J. 2001, A\&A 378, 316

Donati, J.-F., Jardine, M. M., Gregory, S. G., Petit, P., Bouvier, J., Dougados, C., Mnard, F., Cameron, A. C., Harries, T. J., Jeffers, S. V., \& Paletou, F. 2007, MNRAS 380, 1297

Donati, J.-F., Forveille, T., Cameron, A. C., Barnes, J. R., Delfosse, X., Jardine, M. M., \& Valenti, J. A. 2006, Science 311, 633

Donati, J.-F. 1999, MNRAS 302, 457

Donati, J.-F., Semel, M., Carter, B. D., Rees, D. E., \& Collier Cameron, A. 1997, MNRAS 291, 658

Donati, J.-F., Semel, M., Rees, D. E., Taylor, K., \& Robinson, R. D. 1990, A\&6A 232, L1

Elstner, D. \& Korhonen, H. 2005, AN 326, 278 
Engl, H. W., Hanke, M., \& Neubauer, A. 1996, Regularization of Inverse Problems, Kluwer Academic Publishers Group, Dordrecht, The Netherlands

Hussain, G. A. J., van Ballegooijen, A. A., Jardine, M., \& Collier Cameron, A. 2002, ApJ 575, 1078

Kochukhov, O., Bagnulo, S., Wade, G. A., Sangalli, L., Piskunov, N., Landstreet, J. D., Petit, P., \& Sigut, T. A. A. 2004, A\&A 414, 613

Landi Deglinnocenti, E. \& Landi Deglinnocenti, M. 1985, SP 97, 239

Martínez González, M. J., Asensio Ramos, A., Carroll, T. A., Kopf, M., Ramírez Vélez, J. C., \& Semel, M. 2008, A\&A 486, 637

Petit, P., Dintrans, B., Solanki, S. K., Donati, J.-F., Aurire, M., Lignires, F., Morin, J., Paletou, F., Ramirez Velez, J., Catala, C., \& Fares, R. et al. 2008, MNRAS, 388, 80

Piskunov, N. \& Kochukhov, O. 2002, A\&A 381, 736

Press, W. H., Teukolsky, S. A., Vetterling, W. T., \& Flannery, B. P. 1992, Numerical Recipes, Cambridge: University Press, 2nd ed.

Rees, D. E., Durrant, C. J., \& Murphy, G. A. 1989, ApJ 339, 1093

Semel, M. 1989, A\& A 225, 456

Strassmeier, K. G., Woche, M., Andersen, M., \& Ilyin, I. 2007, AN 328, 627

Strassmeier, K. G., Woche, M., Ilyin, I., Popow, E., Bauer, S.-M., Dionies, F., Fechner, T., Weber, M., Hofmann, A., Storm, J., Materne, R., Bittner, W., Bartus, J., Granzer, T., Denker, C., Carroll, T., Kopf, M., DiVarano, I., Beckert, E., Lesser, M. et al. 2008, Groundbased and Airborne Instrumentation for Astronomy, Proc. of the SPIE, Vol. 7014, p. 70140N

Stenflo, J. O. 1994, Solar Magnetic Fields - Polarized Radiation Diagnostics, Astrophysics and Space Science Library, 189, Dordrecht, Boston: Kluwer Academic Publishers

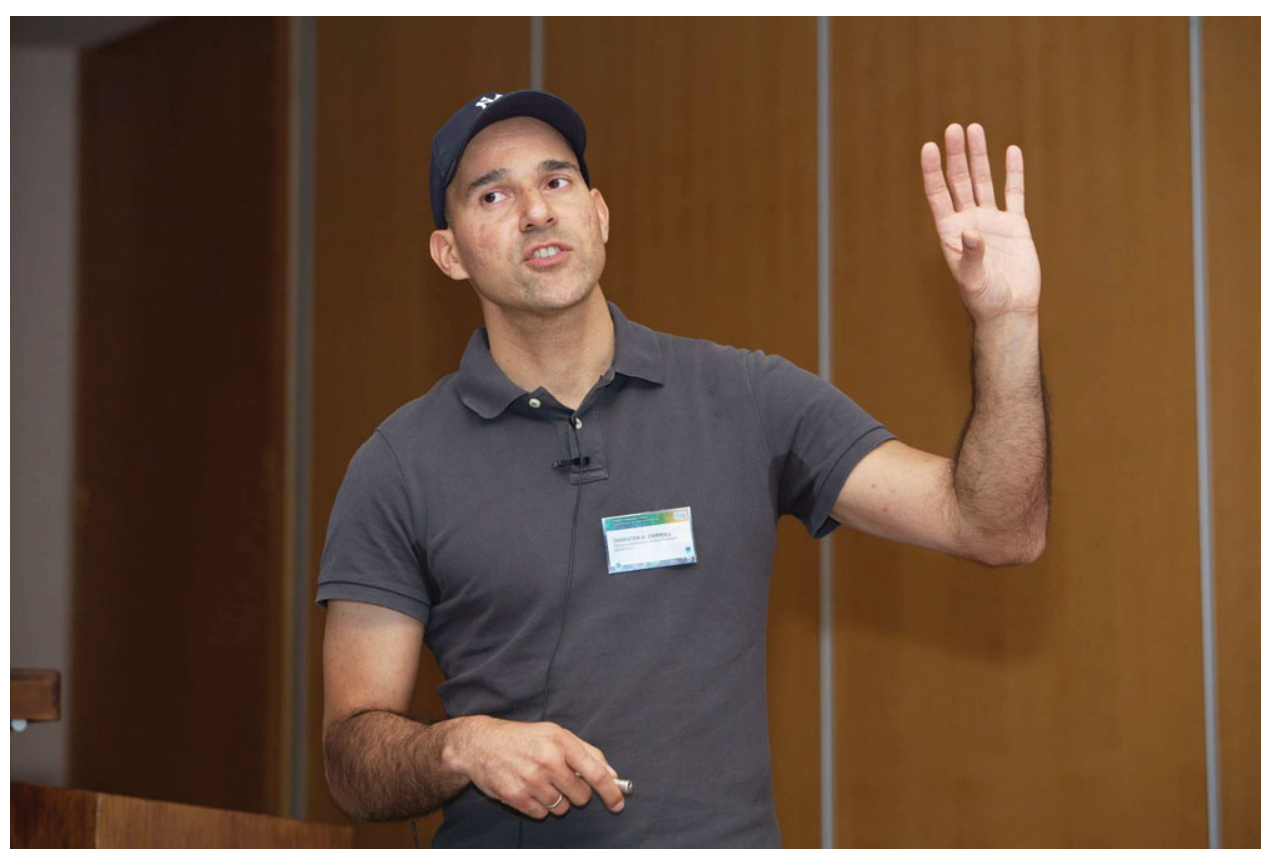

Thorsten Carroll 


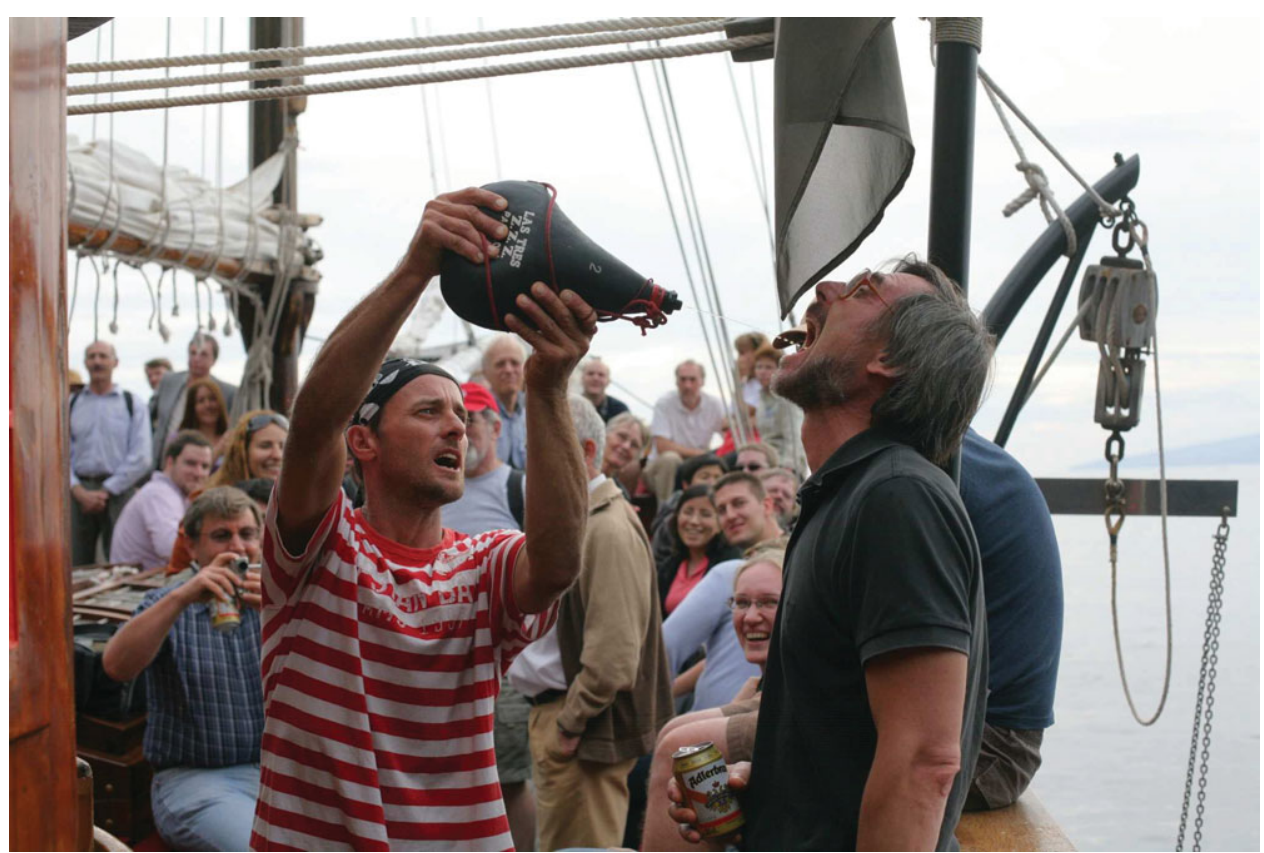

Ilya Ilyin gets special treatment on the pirates ship

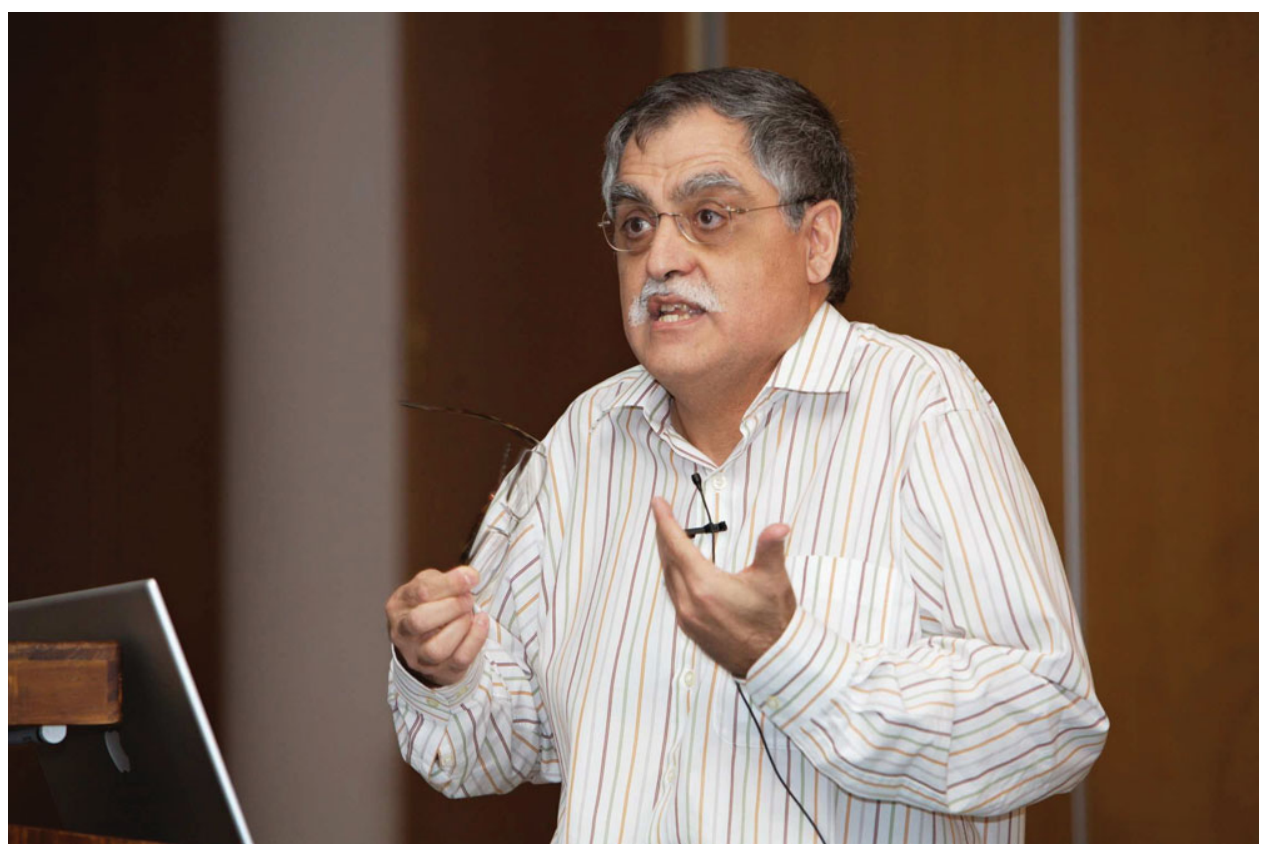

Javier Trujillo Bueno 